\title{
A New Neumann Series Method for Solving a Family of Local Fractional Fredholm and Volterra Integral Equations
}

\author{
Xiao-Jing Ma, ${ }^{1}$ H. M. Srivastava, ${ }^{2}$ Dumitru Baleanu, ${ }^{3,4,5}$ and Xiao-Jun Yang ${ }^{6}$ \\ ${ }^{1}$ College of Electrical Engineering, Xinjiang University, Urumqi, Xinjiang 830046, China \\ ${ }^{2}$ Department of Mathematics and Statistics, University of Victoria, Victoria, BC, Canada V8W 3R 4 \\ ${ }^{3}$ Department of Mathematics and Computer Sciences, Faculty of Arts and Sciences, Cankaya University, 06530 Ankara, Turkey \\ ${ }^{4}$ Department of Chemical and Materials Engineering, Faculty of Engineering, King Abdulaziz University, \\ P.O. Box 80204, Jeddah 21589, Saudi Arabia \\ ${ }^{5}$ Institute of Space Sciences, Magurele, 077125 Bucharest, Romania \\ ${ }^{6}$ Department of Mathematics and Mechanics, China University of Mining and Technology, \\ Xuzhou Campus, Xuzhou, Jiangsu 221008, China
}

Correspondence should be addressed to Xiao-Jing Ma; jingcici@gmail.com

Received 30 May 2013; Accepted 13 June 2013

Academic Editor: J. A. Tenreiro Machado

Copyright (C) 2013 Xiao-Jing Ma et al. This is an open access article distributed under the Creative Commons Attribution License, which permits unrestricted use, distribution, and reproduction in any medium, provided the original work is properly cited.

We propose a new Neumann series method to solve a family of local fractional Fredholm and Volterra integral equations. The integral operator, which is used in our investigation, is of the local fractional integral operator type. Two illustrative examples show the accuracy and the reliability of the obtained results.

\section{Introduction}

Many initial- and boundary-value problems associated with ordinary differential equations (ODEs) and partial differential equations (PDEs) can be transformed into problems of solving the corresponding approximate integral equations. However, some initial- and boundary-value domains are fractal curves, which are everywhere continuous, but nowhere differentiable. As a result, we cannot employ the classical calculus, which requires that the defined functions should be differentiable, in order to process various classes of ordinary differential equations (ODEs) and partial differential equations (PDEs). Applications of fractional calculus, in general, and fractional differential equations [1-10], in particular, as well as various transport phenomena in complex and disordered media and fractional systems, have attracted considerable attention during the past two decades or so [1122].

Recently, local fractional calculus [23-40], processing local fractional continuous non-differential functions, was successfully applied to model the stress-strain relation in fractal elasticity [26, 27], fractal release equation [32], wave equations on Cantor sets [34], fractal heat equation [34], diffusion equation arising in discontinuous heat transfer in fractal media [35], Laplace equation within local fractional operators [36], Schrödinger equation in fractal time-space [37], damped wave equation and dissipative wave equation in fractal strings [38], heat-conduction equation on Cantor sets without heat generation in fractal media [39], and so on. There are some analytical and numerical methods for solving local fractional ODEs and PDEs, such as fractional complex transform method with local fractional operator [35], local fractional variational iteration method [37], Cantor-type cylindrical-coordinate method [38], local fractional Fourier series method [39], local fractional series expansion method [40], Fourier and Laplace transforms with local fractional operator [39], and reference therein.

The Neumann series method was applied to solve the integral equations [41, 42]. Recently, the fractional Neumann series method was considered in $[43,44]$. This paper focuses on a new Neumann series method for solving the local fractional Fredholm and Volterra integral equation being 
here facts in mind. This paper is structured as follows. Section 2 introduces the notations and the basic concepts. Section 3 is devoted to a new Neumann series method via local fractional integral operator. Two illustrative examples are explained in Section 4. Finally, conclusions are reported in Section 5.

\section{Preliminaries}

In order to investigate the local fractional continuity of nondifferential functions, we suggest the result derived from fractal geometry $[34,39]$.

Let $f(x)$ be local fractional continuous on interval $(a, b)$; then we write $[34,35]$

$$
f(x) \in C_{\alpha}(a, b) .
$$

If $f:(F, d) \rightarrow\left(\Omega^{\prime}, d^{\prime}\right)$ is a bi-Lipschitz mapping, then

$$
\rho^{s} H^{s}(F) \leq H^{s}(f(F)) \leq \tau^{s} H^{s}(F)
$$

which leads to

$$
\rho^{\alpha}\left|x_{1}-x_{2}\right|^{\alpha} \leq\left|f\left(x_{1}\right)-f\left(x_{2}\right)\right| \leq \tau^{\alpha}\left|x_{1}-x_{2}\right|^{\alpha},
$$

so that

$$
\left|f\left(x_{1}\right)-f\left(x_{2}\right)\right|<\varepsilon^{\alpha}
$$

where $\rho, \tau>0$ and $x_{1}, x_{2} \in F$.

The result deduced from fractal geometry is related to fractal coarse-grained mass function $\gamma^{\alpha}[F, a, b]$, which reads [34] as

$$
\gamma^{\alpha}[F, a, b]=\frac{H^{\alpha}(F \cap(a, b))}{\Gamma(1+\alpha)},
$$

with

$$
H^{\alpha}(F \cap(a, b))=(b-a)^{\alpha},
$$

where $H^{\alpha}$ is an $\alpha$-dimensional Hausdorff measure.

Notice that we consider that the dimensions of any fractal spaces (e.g., Cantor spaces or the Cantor-like spaces) are a positive numbers. It looks like the Euclidean space because its dimension is also positive number. The detailed results were considered in [34].

For $f(x) \in C_{\alpha}(a, b)$, local fractional integral of $f(x)$ of order $\alpha$ in the interval $[a, b]$ is given by $[34,37,39]$

$$
\begin{aligned}
{ }_{a} I_{b}^{(\alpha)} f(x) & =\frac{1}{\Gamma(1+\alpha)} \int_{a}^{b} f(t)(d t)^{\alpha} \\
& =\frac{1}{\Gamma(1+\alpha)} \lim _{\Delta t \rightarrow 0} \sum_{j=0}^{j=N-1} f\left(t_{j}\right)\left(\Delta t_{j}\right)^{\alpha}
\end{aligned}
$$

where $\Delta t_{j}=t_{j+1}-t_{j}, \Delta t=\max \left\{\Delta t_{1}, \Delta t_{2}, \Delta t_{j}, \ldots\right\}$ and $\left[t_{j}, t_{j+1}\right], j=0, \ldots, N-1, t_{0}=a, t_{N}=b$, is a partition of the interval $[a, b]$.
For any $x \in(a, b)$, we have [34]

$$
{ }_{a} I_{x}^{(\alpha)} f(x)
$$

denoted by

$$
f(x) \in I_{x}^{(\alpha)}(a, b) .
$$

If $f(x) \in I_{x}^{(\alpha)}(a, b)$, then we have [34]

$$
f(x) \in C_{\alpha}(a, b) .
$$

For detailed content of fractal geometrical explanation of local fractional integral, we can see $[34,35]$. Some properties of local fractional integral operator were suggested in (A.1)(A.5).

\section{A New Neumann Series Method to Deal with the Local Fractional Fredholm and Volterra Integral Equations}

In this section, we consider a new Neumann series method to process the local fractional Fredholm and Volterra integral equations.

A new Neumann series method to deal with the local fractional Fredholm integral equation is written in the following form:

$$
u(x)=f(x)+\frac{\lambda^{\alpha}}{\Gamma(1+\alpha)} \int_{a}^{b} K(x, t) u(t)(d t)^{\alpha} .
$$

It is obtained if we set

$$
u_{0}(x)=f(x)
$$

such that

$$
\begin{aligned}
u_{1}(x) & =u_{0}(x)+\frac{\lambda^{\alpha}}{\Gamma(1+\alpha)} \int_{a}^{b} K(x, t) u_{0}(t)(d t)^{\alpha} \\
& =f(x)+\lambda^{\alpha} \psi_{1}(x),
\end{aligned}
$$

where $\psi_{1}(x)=(1 / \Gamma(1+\alpha)) \int_{a}^{b} K(x, t) f(t)(d t)^{\alpha}$.

The zeroth approximation can be written as

$$
\begin{aligned}
u_{2}(x)= & f(x)+\frac{\lambda^{\alpha}}{\Gamma(1+\alpha)} \int_{a}^{b} K(x, t) u_{1}(t)(d t)^{\alpha} \\
= & f(x)+\frac{\lambda^{\alpha}}{\Gamma(1+\alpha)} \\
& \times \int_{a}^{b} K(x, t)\left\{f(x)+\lambda^{\alpha} \psi_{1}(x)\right\}(d t)^{\alpha} \\
= & f(x)+\lambda^{\alpha} \psi_{1}(x)+\lambda^{2 \alpha} \psi_{2}(x),
\end{aligned}
$$

where $\psi_{2}(x)=(1 / \Gamma(1+\alpha)) \int_{a}^{b} K(x, t) \psi_{1}(x)(d t)^{\alpha}$.

Proceeding in this manner, the final solution $u(x)$ can be obtained as

$$
\begin{aligned}
u(x) & =f(x)+\lambda^{\alpha} \psi_{1}(x)+\lambda^{2 \alpha} \psi_{2}(x)+\cdots+\lambda^{n \alpha} \psi_{n}(x)+\cdots \\
& =f(x)+\sum_{n=1}^{\infty} \lambda^{n \alpha} \psi_{n}(x)
\end{aligned}
$$

where $\psi_{n}(x)=(1 / \Gamma(1+\alpha)) \int_{a}^{b} K(x, t) \psi_{n-1}(x)(d t)^{\alpha}, n \geq 1$. 
Now we structure a new Neumann series method to handle the local fractional Volterra integral equation, which reads as

$$
u(x)=f(x)+\frac{\lambda^{\alpha}}{\Gamma(1+\alpha)} \int_{a}^{x} K(x, t) u(t)(d t)^{\alpha} .
$$

The method is applicable provided that $u(x)$ is a local fractional analysis function; that is, $u(x)$ have a local fractional Taylor's expansion around $x=0$.

$u(x)$ can be expressed by a local fractional series expansion; which reads as

$$
u(x)=\sum_{n=1}^{\infty} a_{n} x^{n \alpha}
$$

where the coefficients $a_{n}$ and $x$ are constants that are required to be determined.

We have

$$
\sum_{n=1}^{\infty} a_{n} x^{n \alpha}=f(x)+\frac{\lambda^{\alpha}}{\Gamma(1+\alpha)} \int_{a}^{x} K(x, t) \sum_{n=1}^{\infty} a_{n} x^{n \alpha}(d t)^{\alpha} .
$$

Thus, using a few terms of the expansion in both sides, we find that

$$
\begin{aligned}
a_{0}+a_{1} x^{\alpha}+a_{2} x^{2 \alpha}+\cdots+a_{n} x^{n \alpha}+\cdots \\
=f(x)+\frac{\lambda^{\alpha}}{\Gamma(1+\alpha)} \int_{a}^{x} K(x, t) a_{0}(d t)^{\alpha} \\
\quad+\frac{\lambda^{\alpha}}{\Gamma(1+\alpha)} \int_{a}^{x} K(x, t) a_{1} x^{\alpha}(d t)^{\alpha} \\
\quad+\frac{\lambda^{\alpha}}{\Gamma(1+\alpha)} \int_{a}^{x} K(x, t) a_{2} x^{2 \alpha}(d t)^{\alpha}+\cdots \\
\quad+\frac{\lambda^{\alpha}}{\Gamma(1+\alpha)} \int_{a}^{x} K(x, t) a_{n} x^{n \alpha}(d t)^{\alpha}+\cdots
\end{aligned}
$$

We then write the local fractional Taylor's expansions for $f(x)$ and count the first few integrals in (19). After the integration is performed, we equate the coefficients of the same powers of $x^{\alpha}$ in both sides of (19). By this way, we can determine completely the unknown coefficients and produce solution in a local fractional series form.

\section{Examples}

Example 1. Solve the following local fractional Fredholm integral equation:

$$
u(x)=\Gamma(1+\alpha)+\frac{1}{\Gamma(1+\alpha)} \int_{0}^{1} x^{\alpha} u(t)(d t)^{\alpha} .
$$

Let us consider the zeroth approximation given by

$$
u_{0}(t)=\Gamma(1+\alpha)
$$

The first approximation can be computed as follows:

$$
\begin{aligned}
u_{1}(x) & =\Gamma(1+\alpha)+\frac{1}{\Gamma(1+\alpha)} \int_{0}^{1} x^{\alpha} \Gamma(1+\alpha)(d t)^{\alpha} \\
& =\Gamma(1+\alpha)+x^{\alpha} .
\end{aligned}
$$

Proceeding in this manner, we find the following local fractional series approximation:

$$
\begin{aligned}
u_{2}(x) & =\Gamma(1+\alpha)+\frac{1}{\Gamma(1+\alpha)} \int_{0}^{1} x^{\alpha}\left(\Gamma(1+\alpha)+t^{\alpha}\right)(d t)^{\alpha} \\
& =\Gamma(1+\alpha)+x^{\alpha}\left(1+\frac{\Gamma(1+\alpha)}{\Gamma(1+2 \alpha)}\right) .
\end{aligned}
$$

Similarly, the third approximation reads as follows:

$$
\begin{aligned}
u_{3}(x)= & \Gamma(1+\alpha)+\frac{1}{\Gamma(1+\alpha)} \\
& \times \int_{0}^{1} x^{\alpha}\left(\Gamma(1+\alpha)+t^{\alpha}\left(1+\frac{\Gamma(1+\alpha)}{\Gamma(1+2 \alpha)}\right)\right)(d t)^{\alpha} \\
= & \Gamma(1+\alpha)+x^{\alpha}\left(1+\frac{\Gamma(1+\alpha)}{\Gamma(1+2 \alpha)}+\frac{\Gamma^{2}(1+\alpha)}{\Gamma^{2}(1+2 \alpha)}\right) .
\end{aligned}
$$

The fourth approximation yields

$$
\begin{aligned}
u_{4}(x)= & \Gamma(1+\alpha)+\frac{1}{\Gamma(1+\alpha)} \\
& \times \int_{0}^{1} x^{\alpha}\left(\Gamma(1+\alpha)+t^{\alpha}\right. \\
& \left.\times\left(1+\frac{\Gamma(1+\alpha)}{\Gamma(1+2 \alpha)}+\frac{\Gamma^{2}(1+\alpha)}{\Gamma^{2}(1+2 \alpha)}\right)\right)(d t)^{\alpha} \\
= & \Gamma(1+\alpha)+x^{\alpha}\left(1+\frac{\Gamma(1+\alpha)}{\Gamma(1+2 \alpha)}\right. \\
& \left.+\frac{\Gamma^{2}(1+\alpha)}{\Gamma^{2}(1+2 \alpha)}+\frac{\Gamma^{3}(1+\alpha)}{\Gamma^{3}(1+2 \alpha)}\right) .
\end{aligned}
$$

In conclusion, we get

$$
u_{n}(x)=\Gamma(1+\alpha)+x^{\alpha} \sum_{i=0}^{n}\left(\frac{\Gamma(1+\alpha)}{\Gamma(1+2 \alpha)}\right)^{n} .
$$

Hence,

$$
\begin{aligned}
u(x) & =\lim _{n \rightarrow \infty} u_{n}(x) \\
& =\Gamma(1+\alpha)+x^{\alpha} \lim _{n \rightarrow \infty} \sum_{i=0}^{n}\left(\frac{\Gamma(1+\alpha)}{\Gamma(1+2 \alpha)}\right)^{n} \\
& =\Gamma(1+\alpha)+\frac{x^{\alpha} \Gamma(1+\alpha)}{\Gamma(1+2 \alpha)-\Gamma(1+\alpha)} .
\end{aligned}
$$


Example 2. Obtain the solution of the following local fractional Volterra equation:

$$
u(x)=1+\frac{x^{\alpha}}{\Gamma(1+\alpha)}+\frac{1}{\Gamma(1+\alpha)} \int_{0}^{x} \frac{(t-x)^{\alpha}}{\Gamma(1+\alpha)} u(t)(d t)^{\alpha}
$$

Suppose that there exists the solution in the following local fractional series form:

$$
u(x)=\sum_{n=1}^{\infty} a_{n} x^{n \alpha}
$$

Then, upon substituting the local fractional series into the equation, we find that

$$
\begin{aligned}
& \sum_{n=1}^{\infty} a_{n} x^{n \alpha} \\
& \quad=\frac{x^{\alpha}}{\Gamma(1+\alpha)}+\frac{1}{\Gamma(1+\alpha)} \int_{0}^{x} \frac{(t-x)^{\alpha}}{\Gamma(1+\alpha)} \sum_{n=1}^{\infty} a_{n} x^{n \alpha}(d t)^{\alpha} \\
& =\frac{x^{\alpha}}{\Gamma(1+\alpha)}-\sum_{n=1}^{\infty} \frac{\Gamma(n \alpha+1) a_{n} x^{(n+2) \alpha}}{\Gamma((n+2) \alpha+1)} .
\end{aligned}
$$

Comparing the coefficients of the same powers of $x^{\alpha}$, we get

$$
\begin{gathered}
a_{0}=1, \quad a_{1}=\frac{1}{\Gamma(1+\alpha)}, \\
a_{2}=-\frac{a_{0}}{\Gamma(2 \alpha+1)}, \quad a_{3}=-\frac{\Gamma(\alpha+1) a_{1}}{\Gamma(3 \alpha+1)}, \\
\vdots \\
a_{n}=-\frac{\Gamma((n-2) \alpha+1) a_{n-2}}{\Gamma(n \alpha+1)},
\end{gathered}
$$

and so on. Thus, the values of the coefficients can be calculated as follows:

$$
\begin{gathered}
a_{0}=1, \quad a_{1}=\frac{1}{\Gamma(1+\alpha)}, \\
a_{2}=-\frac{1}{\Gamma(2 \alpha+1)}, \quad a_{3}=-\frac{1}{\Gamma(3 \alpha+1)}, \\
a_{4}=\frac{1}{\Gamma(4 \alpha+1)}, \quad a_{5}=\frac{1}{\Gamma(5 \alpha+1)}, \\
a_{6}=-\frac{1}{\Gamma(6 \alpha+1)}, \quad a_{7}=-\frac{1}{\Gamma(7 \alpha+1)},
\end{gathered}
$$

Hence, the local fractional series solution is given by

$$
\begin{aligned}
u(x)= & \sum_{n=1}^{\infty} a_{n} x^{n \alpha} \\
= & \left(1-\frac{x^{3 \alpha}}{\Gamma(3 \alpha+1)}+\frac{x^{5 \alpha}}{\Gamma(5 \alpha+1)}-\frac{x^{7 \alpha}}{\Gamma(7 \alpha+1)}+\cdots\right) \\
& +\left(\frac{x^{\alpha}}{\Gamma(1+\alpha)}-\frac{x^{2 \alpha}}{\Gamma(2 \alpha+1)}+\frac{x^{4 \alpha}}{\Gamma(4 \alpha+1)}\right. \\
= & \left.-\frac{x^{6 \alpha}}{\Gamma(6 \alpha+1)}+\cdots\right) \\
& \cos _{\alpha} x^{\alpha}+\sin _{\alpha} x^{\alpha}
\end{aligned}
$$

which are satisfied with the condition given by $[34,39]$

$$
E_{\alpha}\left(i^{\alpha} x^{\alpha}\right)=\cos _{\alpha} x^{\alpha}+i^{\alpha} \sin _{\alpha} x^{\alpha},
$$

where the Mittag-Leffler function defined on fractal set of fractal dimension $\alpha$ is suggested by $[34,39]$

$$
E_{\alpha}\left(x^{\alpha}\right)=\sum_{k=0}^{\infty} \frac{x^{\alpha k}}{\Gamma(1+k \alpha)}
$$

\section{Conclusions}

Local fractional differential and integral operators have proven to be useful tools to deal with everywhere continuous (but nowhere differentiable) functions in fractal areas ranging from fundamental science to engineering. In this paper, it is proven that a new Neumann series method can be used for solving the local fractional Fredholm and Volterra integral equations, and their solutions are fractal functions. The proposed method is efficient and leads to accurate, approximately convergent solutions to local fractional Fredholm and Volterra integral equations. It is demonstrated that the solutions of local fractional Fredholm and Volterra integral equations are fractal functions, which are equipped with local fractional continuities. However, the classical and fractional Neumann series methods [41-44] were only applied to continuous functions.

\section{Appendix}

The following properties of local fractional integral operator are valid [34].

(a) For any $f(x) \in C_{\alpha}(a, b), 0<\alpha \leq 1$, we have local fractional multiple integrals, which are written as [34]

$$
x_{0} I_{x}^{(k \alpha)} f(x)=\overbrace{x_{0} I_{x}^{(\alpha)} \cdots x_{0} I_{x}^{(\alpha)}}^{k \text { times }} f(x) \text {. }
$$

(b) If $\psi(x, y) \in C_{\alpha}(a, b) \times C_{\alpha}(c, d)$, then [34]

$$
{ }_{a} I_{b}^{(\alpha)}{ }_{c} I_{b}^{(\alpha)} \psi(x, y)={ }_{c} I_{d}^{(\alpha)}{ }_{a} I_{b}^{(\alpha)} \psi(x, y) .
$$


(c) The sine and cosine subfunctions can, respectively, be written as follows [34, 39]:

$$
\begin{gathered}
\sin _{\alpha} x^{\alpha}=\sum_{k=0}^{\infty}(-1)^{k} \frac{x^{\alpha(2 k+1)}}{\Gamma[1+\alpha(2 k+1)]}, \\
\cos _{\alpha} x^{\alpha}=\sum_{k=0}^{\infty}(-1)^{k} \frac{x^{2 \alpha k}}{\Gamma(1+2 \alpha k)}, \quad 0<\alpha \leq 1 .
\end{gathered}
$$

(d) Suppose that $f(t)$ is local fractional continuous on the interval $[a, b]$. Then

${ }_{a} I_{x}^{(\alpha)}{ }_{a} I_{\tau}^{(\alpha)} f(t)={ }_{a} I_{x}^{(\alpha)} \frac{(x-t)^{\alpha} f(t)}{\Gamma(1+\alpha)} \quad(x \in[a, b])$.

(e) We have

$$
{ }_{0} I_{x}^{(\alpha)}{ }_{0} I_{\tau}^{(\alpha)} \frac{t^{k \alpha}}{\Gamma(k \alpha+1)}=\frac{t^{(k+2) \alpha}}{\Gamma((k+2) \alpha+1)} .
$$

\section{References}

[1] K. B. Oldham and J. Spanier, The Fractional Calculus, Academic Press, London, UK, 1974.

[2] K. S. Miller and B. Ross, An Introduction to the Fractional Calculus and Fractional Differential Equations, John Wiley \& Sons Inc., New York, NY, USA, 1993.

[3] S. G. Samko, A. A. Kilbas, and O. I. Marichev, Fractional Integrals and Derivatives, Gordon and Breach Science Publishers, Yverdon, Switzerland, 1993.

[4] V. Kiryakova, Generalized Fractional Calculus and Applications, vol. 301, Longman Scientific \& Technical, Harlow, UK, 1994.

[5] I. Podlubny, Fractional Differential Equations, vol. 198, Academic Press, San Diego, Calif, USA, 1999.

[6] R. Hilfer, Ed., Applications of Fractional Calculus in Physics, World Scientific Publishing, River Edge, NJ, USA, 2000.

[7] A. A. Kilbas, H. M. Srivastava, and J. J. Trujillo, Theory and Applications of Fractional Differential Equations, vol. 204, Elsevier Science B.V., Amsterdam, The Netherlands, 2006.

[8] J. Sabatier, O. P. Agrawal, and J. A. Tenreiro Machado, Advances in Fractional Calculus: Theoretical Developments and Applications in Physics and Engineering, Springer, New York, NY, USA, 2007.

[9] F. Mainardi, Fractional Calculus and Waves in Linear Viscoelasticity, Imperial College Press, London, UK, 2010.

[10] G. M. Zaslavsky, "Chaos, fractional kinetics, and anomalous transport," Physics Reports, vol. 371, no. 6, pp. 461-580, 2002.

[11] J. Hristov, "Heat-balance integral to fractional (half-time) heat diffusion sub-model," Thermal Science, vol. 14, no. 2, pp. 291-316, 2010.

[12] B. J. West, M. Bologna, and P. Grigolini, Physics of Fractal Operators, Springer, New York, NY, USA, 2003.

[13] R. Metzler and J. Klafter, "The restaurant at the end of the random walk: recent developments in the description of anomalous transport by fractional dynamics," Journal of Physics A, vol. 37, no. 31, pp. R161-R208, 2004.

[14] L. M. Zelenyǐ and A. V. Milovanov, "Fractal topology and strange kinetics: from percolation theory to problems in cosmic electrodynamics," Physics-Uspekhi, vol. 47, no. 8, pp. 749-788, 2004.
[15] G. M. Zaslavsky, Hamiltonian Chaos and Fractional Dynamics, Oxford University Press, Oxford, UK, 2008.

[16] J. A. T. Machado, "Analysis and design of fractional-order digital control systems," Systems Analysis Modelling Simulation, vol. 27, no. 2-3, pp. 107-122, 1997.

[17] J. A. T. Machado, "Fractional-order derivative approximations in discrete-time control systems," Systems Analysis Modelling Simulation, vol. 34, no. 4, pp. 419-434, 1999.

[18] R. Herrmann, Fractional Calculus: An Introduction for Physicists, World Scientific, 2011.

[19] J. Klafter, S. C. Lim, and R. Metzler, Fractional Dynamics: Recent Advances, World Scientific, 2012.

[20] M. D. Ortigueira, Fractional Calculus for Scientists and Engineers, Springer, 2011.

[21] D. Baleanu, K. Diethelm, E. Scalas, and J. J. Trujillo, Fractional Calculus Models and Numerical Methods, Complexity, Nonlinearity and Chaos, World Scientific, Boston, Mass, USA, 2012.

[22] I. Petras, Fractional-Order Nonlinear Systems: Modeling. Analysis and Simulation, Springer, Berlin, Germany, 2011.

[23] K. M. Kolwankar and A. D. Gangal, "Fractional differentiability of nowhere differentiable functions and dimensions," Chaos, vol. 6, no. 4, pp. 505-513, 1996.

[24] K. M. Kolwankar and A. D. Gangal, "Local fractional FokkerPlanck equation,” Physical Review Letters, vol. 80, pp. 214-217, 1998.

[25] F. B. Adda and J. Cresson, "About non-differentiable functions," Journal of Mathematical Analysis and Applications, vol. 263, no. 2, pp. 721-737, 2001.

[26] A. Carpinteri, B. Chiaia, and P. Cornetti, "Static-kinematic duality and the principle of virtual work in the mechanics of fractal media," Computer Methods in Applied Mechanics and Engineering, vol. 191, no. 1-2, pp. 3-19, 2001.

[27] A. Carpinteri, B. Chiaia, and P. Cornetti, "The elastic problem for fractal media: basic theory and finite element formulation," Computers and Structures, vol. 82, no. 6, pp. 499-508, 2004.

[28] Y. Chen, Y. Yan, and K. Zhang, "On the local fractional derivative," Journal of Mathematical Analysis and Applications, vol. 362, no. 1, pp. 17-33, 2010.

[29] A. Babakhani and V. D. Gejji, "On calculus of local fractional derivatives," Journal of Mathematical Analysis and Applications, vol. 270, no. 1, pp. 66-79, 2002.

[30] G. Jumarie, "Table of some basic fractional calculus formulae derived from a modified Riemann-Liouville derivative for nondifferentiable functions," Applied Mathematics Letters, vol. 22, no. 3, pp. 378-385, 2009.

[31] G. Jumarie, "Probability calculus of fractional order and fractional Taylor's series application to Fokker-Planck equation and information of non-random functions," Chaos, Solitons and Fractals, vol. 40, no. 3, pp. 1428-1448, 2009.

[32] W. Chen, H. Sun, X. Zhang, and D. Korošak, "Anomalous diffusion modeling by fractal and fractional derivatives," Computers and Mathematics with Applications, vol. 59, no. 5, pp. 1754-1758, 2010.

[33] W. Chen, "Time-space fabric underlying anomalous diffusion," Chaos, Solitons and Fractals, vol. 28, no. 4, pp. 923-925, 2006.

[34] X.-J. Yang, Advanced Local Fractional Calculus and Its Applications, World Science, New York, NY, USA, 2012.

[35] M.-S. Hu, D. Baleanu, and X.-J. Yang, "One-phase problems for discontinuous heat transfer in fractal media," Mathematical Problems in Engineering, vol. 2013, Article ID 358473, 3 pages, 2013. 
[36] A. Liangprom and K. Nonlaopon, "On the convolution equation related to the diamond Klein-Gordon operator," Abstract and Applied Analysis, vol. 2011, Article ID 908491, 16 pages, 2011.

[37] W.-H. Su, D. Baleanu, X.-J. Yang, and H. Jafari, "Damped wave equation and dissipative wave equation in fractal strings within the local fractional variational iteration method," Fixed Point Theory and Applications, vol. 2013, no. 1, pp. 1-11, 2013.

[38] X.-J. Yang, H. M. Srivastava, J.-H. He, and D. Baleanu, "Cantortype cylindrical-coordinate method for differential equations with local fractional derivatives," Physics Letters A, vol. 377, no. 38-30, pp. 1696-1700.

[39] X.-J. Yang, Local Fractional Functional Analysis and Its Applications, Asian Academic, Hong Kong, China, 2011.

[40] A.-M. Yang, X.-J. Yang, and Z.-B. Li, "Local fractional series expansion method for solving wave and diffusion equations on Cantor sets," Abstract and Applied Analysis, vol. 2013, Article ID 351057, 5 pages, 2013.

[41] D. Medková, "On the convergence of Neumann series for noncompact operators," Czechoslovak Mathematical Journal, vol. 41, no. 2, pp. 312-316, 1991.

[42] P. Stefanov and G. Uhlmann, "Thermoacoustic tomography with variable sound speed," Inverse Problems, vol. 25, no. 7, Article ID 075011, 2009.

[43] H. M. Srivastava and R. K. Saxena, "Operators of fractional integration and their applications," Applied Mathematics and Computation, vol. 118, no. 1, pp. 1-52, 2001.

[44] A. Ebaid, D. M. M. ElSayed, and M. D. Aljoufi, "Fractional calculus model for damped Mathieu equation: approximate analytical solution," Applied Mathematical Sciences, vol. 6, no. 81-84, pp. 4075-4080, 2012. 


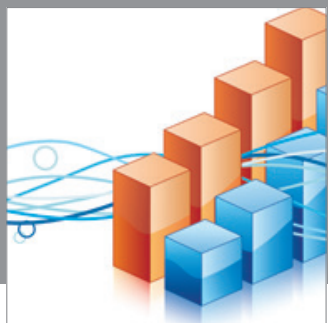

Advances in

Operations Research

mansans

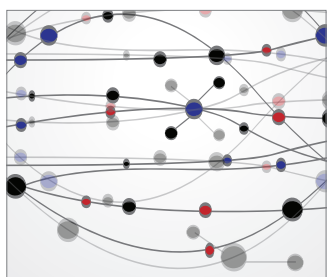

The Scientific World Journal
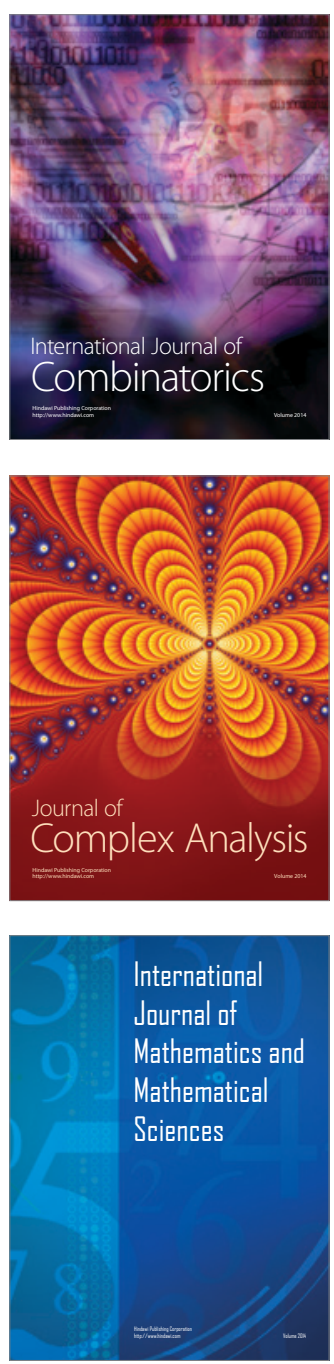
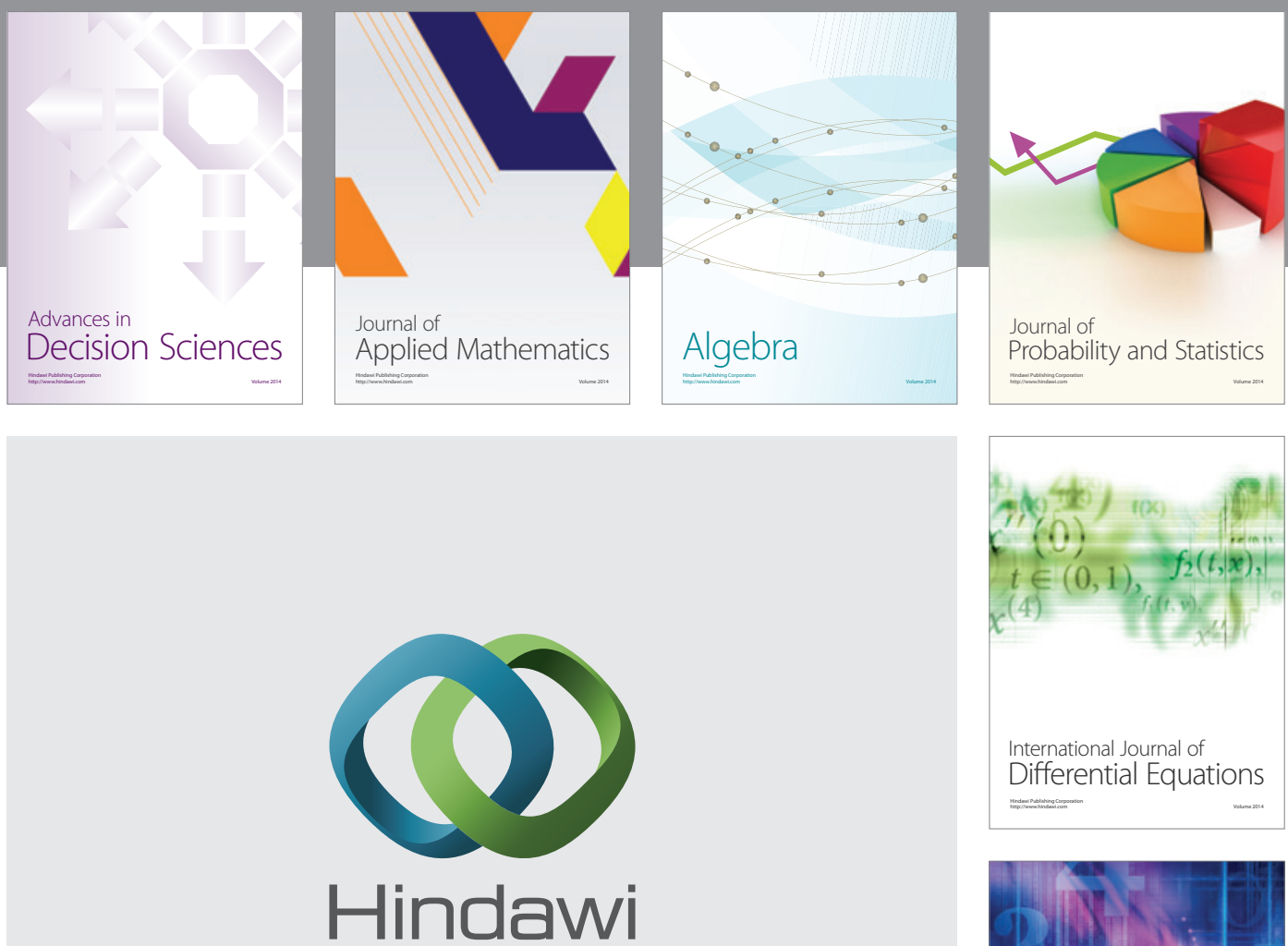

Submit your manuscripts at http://www.hindawi.com
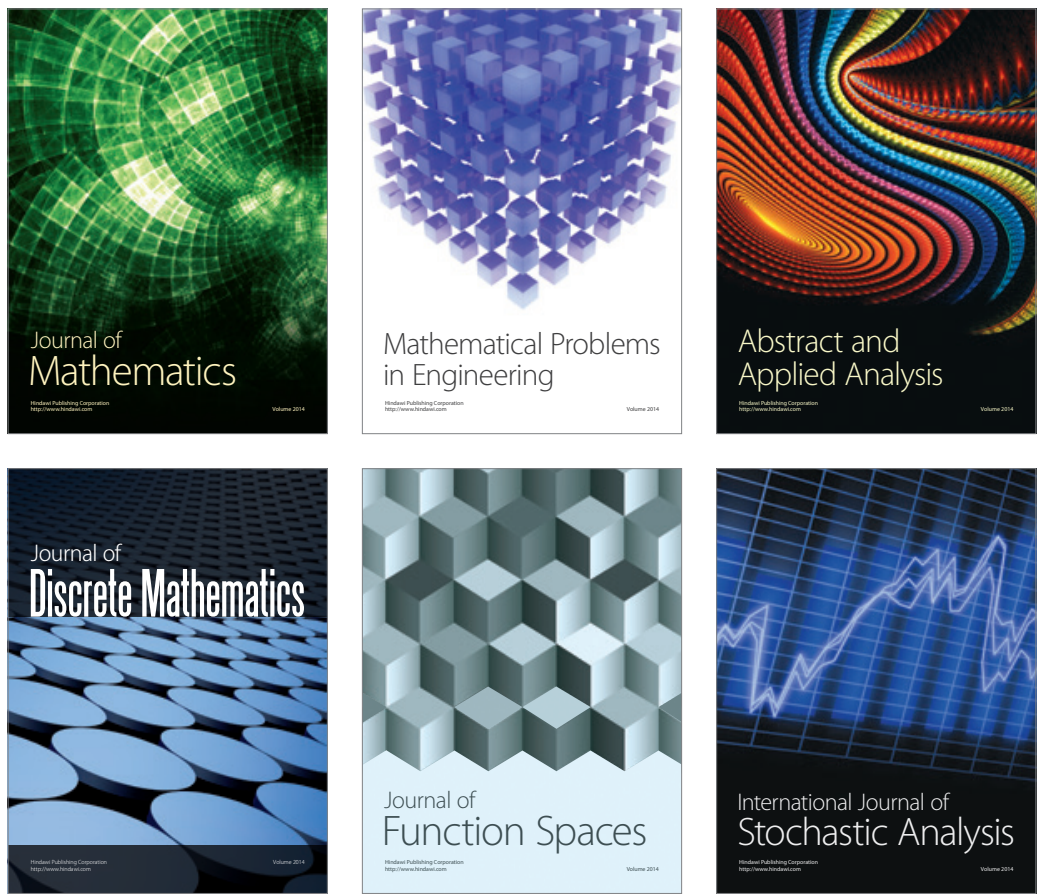

Journal of

Function Spaces

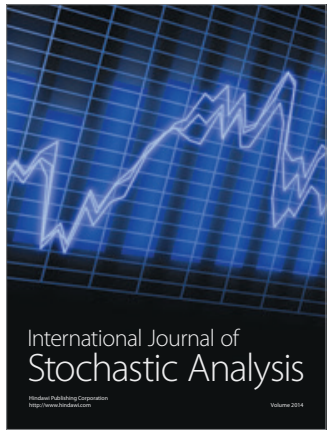

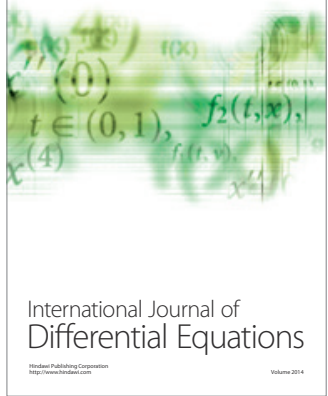
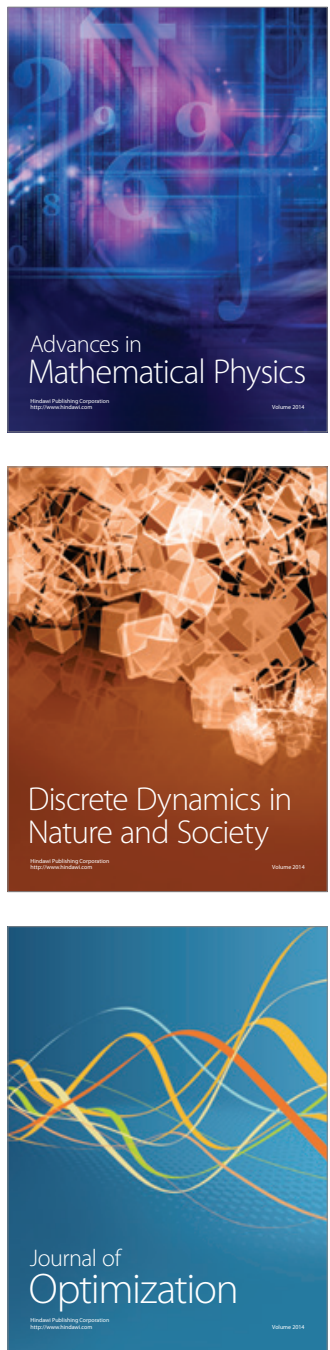